\title{
Carcinoma gástrico e disfagia: uma revisão sistemática
}

\author{
Gastric carcinoma and dysfunction: \\ a systematic review
}

\section{Carcinoma gástrico y disfagia: una revisión sistemática}

\author{
Eduarda Besen* \\ Claudia Tiemi Mituuti* \\ Emanuelle Moreira* \\ Deivid de Souza Silveira* \\ Patrícia Haas*
}

\section{Resumo}

Introdução: O carcinoma gástrico, também conhecido como câncer de estômago é um dos tipos de câncer com grande incidência no Brasil, segundo dados do INCA. As formas de tratamento para carcinomas gástricos estão relacionadas ao estágio em que a doença se encontra, podem variar de cirurgias, ressecções a gastrectomias e linfadenectomias, podendo ser necessária a presença de quimioterapia. Sabe-se que alguns dos muitos tipos de cânceres podem levar à apresentação de algum quadro de disfagia, caracterizada pelas alterações no processo de deglutição. Objetivo: verificar qual o impacto do câncer gástrico na disfagia. Material e Método: Buscas de artigos nas bases de dados Medline (Pubmed), Cochrane Library, SciELO, LILACS e MEDLINE sem restrição de localização ou idiomas, publicados no período de janeiro de 2010 até agosto de 2018. A pesquisa foi realizada na base de dados com os descritores ("Deglutition Disorders" or "Dysphagia" or "Esophageal Dysphagia" or "Oropharyngeal Dysphagia" or "Swallowing Disorders") and ("Stomach Neoplasms" or "Cancer of Stomach" or "Cancer of the Stomach" or "Gastric Cancer" or "Gastric Cancer, Familial Diffuse" or "Gastric Neoplasms" or "Neoplasms, Gastric or Neoplasms", "Stomach" or "Stomach Cancer"). Resultados: A relação entre o câncer gástrico e a disfagia é abordada como uma importante consequência dos tipos de tratamentos. Foram encontrados cinco estudos que

* Universidade Federal de Santa Catarina, Florianópolis, Santa Catarina, Brasil.

Contribuição dos autores:

CTM e EM: concepção e delineamento do estudo, aquisição de dados, na escrita do artigo, revisão crítica do conteúdo intelectual relevante e aprovação final da versão a ser submetida;

DSS: concepção e delineamento do estudo, análise e interpretação de dados, na escrita do artigo, revisão crítica do conteúdo intelectual relevante e aprovação final da versão a ser submetida.

PH: contribuição substancial na concepção e delineamento do estudo, na escrita do artigo e aprovação final da versão a ser submetida.

E-mail para correspondência: Eduarda Besen - dudabesen@gmail.com

Recebido: 04/05/2019

Aprovado: 03/03/2020 
responderam a pergunta norteadora. Três dos cinco estudos selecionados tratam da qualidade de vida dos pacientes posteriormente ao tratamento. Conclusão: Foi verificado que a disfagia é observada como uma possível consequência do carcinoma gástrico e seu tratamento.

Palavras-chave: Transtornos de Deglutição; Neoplasias Gástricas; Qualidade de Vida.

\section{Abstract}

Introduction: Gastric carcinoma, also known as stomach cancer, is one of the types of cancer with great incidence in Brazil, according to INCA data. The forms of treatment for gastric carcinomas are related to the stage in which the disease is, may vary from surgeries, resections to gastrectomies and lymphadenectomies, and the presence of chemotherapy may be necessary. It is known that some of the many types of cancers can lead to the presentation of some dysphagia, characterized by changes in the swallowing process. Objective: to verify the impact of gastric cancer on dysphagia. Material and Method: Searches for articles in the Medline (Pubmed), Cochrane Library, SciELO, LILACS and MEDLINE databases without restrictions on location or languages, published between January 2010 and August 2018. The research was carried out on the basis of data with descriptors ("Deglutition Disorders" or "Dysphagia" or "Esophageal Dysphagia" or "Oropharyngeal Dysphagia" or "Swallowing Disorders") and ("Stomach Neoplasms" or "Cancer of Stomach" or "Cancer of the Stomach" or " Gastric Cancer "or" Gastric Cancer, Familial Diffuse "or" Gastric Neoplasms "or" Neoplasms, Gastric or Neoplasms ", "Stomach "or" Stomach Cancer "). Results: The relationship between gastric cancer and dysphagia is addressed as an important consequence of the types of treatments. Five studies were found that answered the guiding question. Three of the five selected studies deal with patients' quality of life after treatment. Conclusion: It was found that dysphagia is seen as a consequence of gastric carcinoma and its treatment.

Keywords: Deglutition Disorders; Stomach Neoplasm; Quality of life.

\section{Resumen}

Introducción: el carcinoma gástrico, también conocido como cáncer de estómago, es uno de los tipos de cáncer con gran incidencia en Brasil, según datos de INCA. Las formas de tratamiento para los carcinomas gástricos están relacionadas con la etapa en que se encuentra la enfermedad, pueden variar de cirugías, resecciones a gastrectomías y linfadenectomías, y puede ser necesaria la presencia de quimioterapia. Se sabe que algunos de los muchos tipos de cáncer pueden conducir a la presentación de cierta disfagia, caracterizada por cambios en el proceso de deglución. Objetivo: verificar el impacto del cáncer gástrico en la disfagia. Material y método: búsquedas de artículos en las bases de datos Medline (Pubmed), Cochrane Library, SciELO, LILACS y MEDLINE sin restricciones de ubicación o idiomas, publicadas entre enero de 2010 y agosto de 2018. La investigación se realizó sobre la base de datos con descriptores ("Trastornos de la deglución" o "Disfagia" o "Disfagia esofágica" o "Disfagia orofaríngea" o "Trastornos de la deglución") y ("Neoplasias estomacales" o "Cáncer de estómago" o "Cáncer de estómago" o " Cáncer gástrico "o" Cáncer gástrico, difuso familiar "o" Neoplasias gástricas "o" Neoplasias, gástricas o neoplasias "," Estómago "o" Cáncer de estómago "). Resultados: la relación entre el cáncer gástrico y la disfagia se aborda como una consecuencia importante de los tipos de tratamientos. Se encontraron cinco estudios que respondieron a la pregunta guía. Tres de los cinco estudios seleccionados abordan la calidad de vida de los pacientes después del tratamiento. Conclusión: se encontró que la disfagia se considera una posible consecuencia del carcinoma gástrico y su tratamiento.

Palabras clave: Trastornos de Deglución; Neoplasias Gástricas; Calidad de Vida. 


\section{Introdução}

O carcinoma gástrico, também conhecido como câncer de estômago é um dos tipos de câncer com grande incidência no Brasil, segundo dados do Instituto Nacional de Câncer José Alencar Gomes da Silva (INCA). O câncer de estômago ocupa o terceiro lugar no índice de maior incidência em homens e o quinto lugar no caso das mulheres. A estimativa para o ano de 2018 foi de 21.290 novos casos, sendo 13.540 em pessoas do sexo masculino e 7.750 de sexo feminino ${ }^{1,2}$.

Apesar de apresentar uma diminuição nos casos, o carcinoma gástrico ainda é o quinto tipo de câncer mais comum em todo o mundo, além disso, nos países do ocidente grande parte dos pacientes tem um diagnóstico tardio, o que compromete a efetividade do tratamento e aumenta o risco de recaídas após cirurgia de intenções curativas ${ }^{3}$. $\mathrm{O}$ carcinoma gástrico pode ter origem endógena (genética) ou exógena, relacionada ao ambiente. Um dos agentes externos mais relacionados é a Helicobacter pylori, gerador de infecções que podem gerar ao paciente agravo em inflamações já presentes, como a úlcera e a gastrite, evoluindo-as a tumores. Além dos fatores hereditários e uso e abuso de substâncias como o fumo e o álcool ${ }^{4,2}$.

A questão nutricional do indivíduo está diretamente relacionada com o surgimento do tumor, desta forma a nutrição do paciente é vista como um fator exógeno, tendo em vista que o alimento pode transformar a mucosa gástrica, propiciando o surgimento da neoplasia. A nutrição é considerada um fator importante durante o tratamento, podendo afetar diretamente os resultados do mesmo ${ }^{5,6}$

As formas de escolha para o tratamento de carcinomas gástricos estão relacionadas ao estágio em que a doença se encontra e podem variar de cirurgias, ressecções a gastrectomias e linfadenectomias à quimioterapia. Na maior parte dos casos o tumor é descoberto em seu estágio mais avançado diminuindo as possibilidades de tratamento e de cura ${ }^{7,8}$. O tratamento mais comum e eficaz tem sido a cirurgia. Realiza-se a retirada da área afetada pelo tumor, ou seja, há uma diminuição do espaço da câmara gástrica, ou ainda a ressecção cirúrgica total, mudando desta forma o funcionamento normal do órgão que deve passar por uma fase de adaptação impactante no processo de nutrição do paciente $^{9,10,11}$.
Como consequência de alguns tipos de cânceres, o indivíduo afetado pode apresentar alguma alteração na deglutição, ou seja, algum quadro de disfagia. A deglutição é uma ação neuromotora muito complexa e tem como objetivo transportar o alimento da boca até o estômago visando à nutrição e hidratação do indivíduo. Salienta-se que qualquer tipo de alteração em alguma fase deste processo pode ser chamado de disfagia ${ }^{12,13}$. A deglutição possui quatro fases a serem observadas, como a fase preparatória, oral, faríngea e esofágica. As fases oral e preparatória são desenvolvidas voluntariamente, enquanto a fase faríngea e esofágica são efetuadas de forma involuntária a partir de controle neurológico, onde o estômago deve participar ${ }^{14,15}$. A disfagia pode ter origem mecânica, neurogênica, senil ou psicogênica, podendo gerar grande impacto na vida do paciente e assim, comprometer sua nutrição, hidratação, funções pulmonares ou até convívio social, e no caso do paciente já hospitalizado pode agravar o quadro clínico resultando a óbito $16,17,18,19$.

O conhecimento científico, sobre os motivos que levam indivíduos portadores de câncer gástrico desenvolver disfagia tem sido discutido, bem como em qual fase do câncer (pré, durante e/ou após tratamento) encontra-se com maior ocorrência a disfagia, além de compreender o impacto do tratamento escolhido para cada indivíduo. Esta pesquisa sugere uma maior importância no cuidado da qualidade de vida dos pacientes.

A presente pesquisa apresenta como objetivo principal verificar evidências científicas sobre a relação entre o câncer de estômago e a disfagia, visando responder a seguinte pergunta norteadora: Qual o impacto do câncer gástrico na disfagia?

\section{Métodos}

\section{Caracterização da pesquisa e estratégias de busca}

A revisão sistemática foi conduzida conforme as recomendações do Preferred Reporting Intens for Systematic Reviews and Meta-Analyses (PRISMA) ${ }^{20}$.

As buscas por artigos científicos foram conduzidas por dois pesquisadores independentes nas bases de dados eletrônicas Medline (Pubmed), Cochrane Library, SciELO, LILACS e MEDLINE sem restrição de localização ou idiomas, publicados no período de janeiro de 2010 até agosto de 2018. 
A pesquisa foi estruturada e organizada na forma PICO, que representa um acrônimo para População alvo, a Intervenção, Comparação e "Outcomes" (desfechos).

Os descritores foram selecionados a partir do dicionário Descritores em Ciências da Saúde (DeCS) Medical Subject Heading Terms (MeSH), haja vista a sua grande utilização pela comunidade científica para a indexação de artigos na base de dados PubMed. Diante os descritores, foi realizada a adequação para as outras bases utilizadas. Em um primeiro momento foram propostas para as buscas as seguintes palavras-chave e operadores boleanos: ("Deglutition Disorders" or "Dysphagia" or "Esophageal Dysphagia" or "Oropharyngeal Dysphagia" or "Swallowing Disorders") and ("Stomach Neoplasms" or "Cancer of Stomach" or "Cancer of the Stomach" or "Gastric Cancer" or "Gastric Cancer, Familial Diffuse" or "Gastric Neoplasms" or "Neoplasms, Gastric or Neoplasms", "Stomach" or "Stomach Cancer").

\section{Critérios de elegibilidade}

Critérios de inclusão

Os desenhos dos estudos selecionados foram relatos de casos, estudos de casos e controle, ensaios clínicos controlados, estudos de coorte, estudos em triagem, estudos observacionais e estudos randomizados. Estudos em idiomas em português, inglês e espanhol foram incluídos. Período incluído de 2010 a 2018 (Quadro 1).

\section{Critérios de exclusão}

Foram excluídos estudos publicados no formato de Cartas ao editor, diretrizes, revisões sistemáticas, meta análises e resumos. Foram excluídos artigos publicados anteriormente a 2010. Estudos que não tenham descrito ou que foram pouco claros ou indisponíveis representados no Quadro 1.

Quadro 1. Critérios de inclusão e exclusão.

\begin{tabular}{|l|l|}
\hline Critérios de Inclusão & $\begin{array}{l}\text { Estudos de caso, estudos longitudinais e estudos clínicos randomizados ou } \\
\text { não randomizados com intervenções }\end{array}$ \\
\hline Delineamento & $\begin{array}{l}\bullet \quad \text { Disfagia } \\
\text { Pacientes }\end{array}$ \\
\hline Localização & sem restrição \\
\hline Idioma & sem restrição \\
\hline Critérios de Exclusão & Estudos de prevalência, intervenções feitas por outros profissionais \\
\hline Delineamento & Estudos pouco claros, mal descritos ou inadequados \\
\hline Estudos & Somente em resumos \\
\hline Forma de publicação &
\end{tabular}

Seleção dos estudos

A seleção dos estudos foi realizada por dois examinadores independentes. Inicialmente foram excluídos estudos duplicados, após, baseados no título, em seguida, os resumos foram analisados e apenas os que foram potencialmente elegíveis foram selecionados para avaliação na íntegra. As divergências foram resolvidas por consenso entre os autores e quando necessário consultado o terceiro pesquisador.

\section{Extração de dados}

A extração dos dados para o processo de elegibilidade dos estudos foi realizada utilizando-se uma ficha elaborada pelos pesquisadores em Pro- grama Excel $^{\circledR}$, na qual os dados extraídos foram adicionados inicialmente por um dos pesquisadores e então conferidos pelo outro pesquisador. Para os dados obtidos dos estudos elegíveis, estes também foram transportados para uma planilha em mesmo programa, a fim de organizar os resultados.

\section{Resultados}

Um total de 268 artigos foi identificado nas buscas primárias (Figura 1). No entanto, ao realizar a exclusão por repetição restaram 258 artigos, e após exclusão por título e abstracts restaram 244 artigos. Assim, 14 artigos foram selecionados 
para a avaliação em conformidade com o título e seus resumos revisados. Ao avaliar os artigos na totalidade, cinco preencheram todos os critérios de inclusão propostos os quais atenderam à pergunta norteadora. Esta seleção de inclusão foi estabelecida conforme as recomendações do Preferred Reporting Intens for Systematic Reviews and Meta-Analyses (PRISMA).

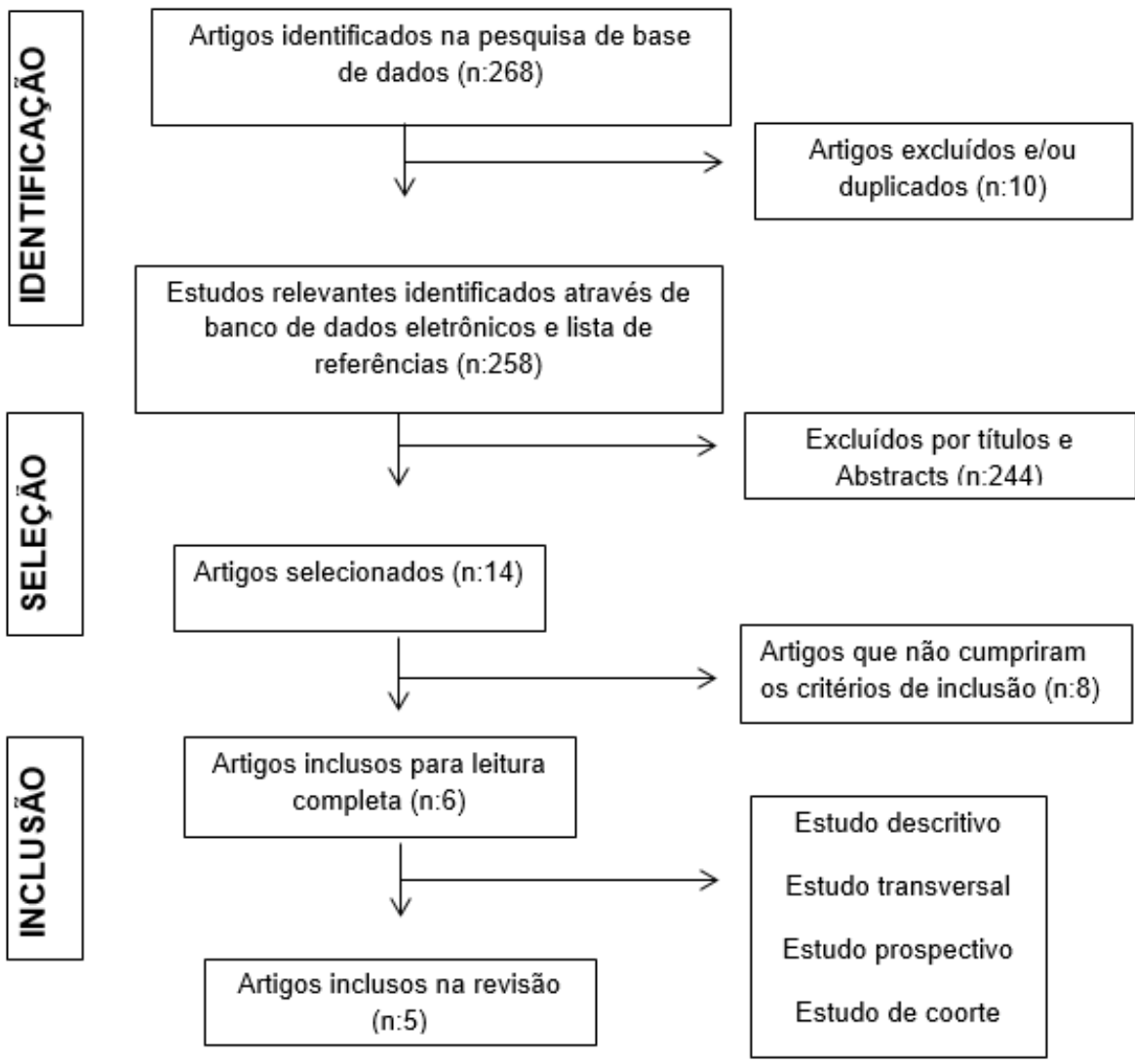

Figura 1. Fluxograma de busca e refinamento dos artigos.

Verificada a qualidade metodológica, assinalou-se a pontuação obtida, por meio de um protocolo para pontuação qualitativa dos estudos. Os estudos incluídos obtiveram pontuação $\geq$ a 11 pontos. Esta pontuação reflete que a qualidade dos estudos incluídos na presente revisão sistemática atingiram alta qualidade.
No Quadro 2, foram descritos os resultados dos artigos incluídos nesta pesquisa. Salienta-se que todos os estudos que cumpriram os critérios de inclusão foram publicados nos últimos seis anos, ou seja, no período de 2014 a 2016, ressaltando ser este um importante assunto discutido pela comunidade científica devido a sua relevância. Dos cinco artigos incluídos nesta revisão científica, três são da Coréia do Sul, um da Irlanda e o outro da Suécia. 
Quadro 2. Síntese dos artigos inclusos.

\begin{tabular}{|c|c|c|c|c|c|}
\hline Autor/ano & Local & Objetivo & Amostra & Métodos & Resultados \\
\hline $\begin{array}{l}\text { PARK, Ji } \\
\text { Yeon et al. } \\
\text { (2014) }\end{array}$ & $\begin{array}{l}\text { Coreia } \\
\text { do Sul }\end{array}$ & $\begin{array}{c}\text { Qualidade de } \\
\text { vida após câncer } \\
\text { gástrico. }\end{array}$ & $N=30$ & $\begin{array}{l}\text { Foi utilizado os questionários } \\
\text { (EORTC) (QLQ-C30) e o } \\
\text { módulo específico do câncer } \\
\text { gástrico (QLQ-STO22) antes } \\
\text { da operação e após no 1, } \\
3,6 \text { e } 12 \text { meses de pós- } \\
\text { cirurgia. Os dados de QVRS } \\
\text { dos pacientes incluídos } \\
\text { foram comparados com os } \\
\text { valores de referência obtidos } \\
\text { na população geral. }\end{array}$ & $\begin{array}{l}\text { Os piores escores para a } \\
\text { maioria dos domínios foram } \\
\text { observados em uma semana } \\
\text { pós-cirurgia e geralmente } \\
\text { retornaram aos níveis basais } \\
\text { dentro de } 3 \text { meses, exceto } \\
\text { por fadiga, disfagia, dor e } \\
\text { restrição alimentar. }\end{array}$ \\
\hline $\begin{array}{l}\text { PARK, Jung- } \\
\text { hoon et al. } \\
(2014)\end{array}$ & $\begin{array}{l}\text { Coreia } \\
\text { do Sul }\end{array}$ & $\begin{array}{l}\text { Colocação de } \\
\text { stents metálicos } \\
\text { em pacientes } \\
\text { com obstrução } \\
\text { maligna } \\
\text { recorrente } \\
\text { no estômago } \\
\text { cirurgicamente } \\
\text { alterado. }\end{array}$ & $N=196$ & $\begin{array}{c}\text { Pacientes tratados com } \\
\text { cinco diferentes tipos de } \\
\text { cirurgia gástrica realizada } \\
\text { para o câncer gástrico. } \\
\text { Sendo avaliado os índices } \\
\text { de sucesso técnico e clínico, } \\
\text { complicações, escore de } \\
\text { disfagia e influência da } \\
\text { quimioterapia. }\end{array}$ & $\begin{array}{c}\text { A colocação de stents } \\
\text { foi tecnicamente bem- } \\
\text { sucedida melhorando o } \\
\text { escore médio de disfagia. } \\
\text { E a quimioterapia foi } \\
\text { significativamente associada } \\
\text { a um aumento da sobrevida. }\end{array}$ \\
\hline $\begin{array}{l}\text { SUNDE, B. } \\
\text { et al. (2016) }\end{array}$ & Suécia & $\begin{array}{c}\text { Alívio da } \\
\text { disfagia durante } \\
\text { o tratamento } \\
\text { neoadjuvante } \\
\text { para câncer } \\
\text { de esôfago } \\
\text { ou junção } \\
\text { gastroesofágica }\end{array}$ & $N=25$ & $\begin{array}{c}\text { Pacientes agendados para } \\
\text { terapia neoadjuvante foram } \\
\text { recrutados e avaliados } \\
\text { quanto à disfagia e apetite } \\
\text { no início do estudo, } \\
\text { após o primeiro ciclo de } \\
\text { tratamento pré-operatório } \\
\text { com quimioterapia ou } \\
\text { quimiorradioterapia e antes } \\
\text { da cirurgia. Foi utilizado } \\
\text { o escores de disfagia de } \\
\text { Watson e Ogilvie para avaliar } \\
\text { a disfagi e o questionário } \\
\text { de escala visual-apetite } \\
\text { de Edmonton Assessment } \\
\text { System para avaliação do } \\
\text { apetite. }\end{array}$ & $\begin{array}{l}\text { Não foi possível demonstrar } \\
\text { qualquer associação entre } \\
\text { o alívio da disfagia e o grau } \\
\text { de resposta histológica à } \\
\text { terapia neoadjuvante na } \\
\text { peça cirúrgica. Mostra que a } \\
\text { quimioterapia neoadjuvante } \\
\text { à base de platina - 5FU, } \\
\text { com ou sem radioterapia } \\
\text { concomitante, efetiva e } \\
\text { prontamente alivia a disfagia } \\
\text { em pacientes com câncer } \\
\text { de esôfago ou junção } \\
\text { gastroesofágica já após o } \\
\text { primeiro ciclo. }\end{array}$ \\
\hline $\begin{array}{l}\text { Goh YM, et } \\
\text { al. (2015) }\end{array}$ & Irlanda & $\begin{array}{l}\text { Qualidade } \\
\text { de vida após } \\
\text { carcinoma } \\
\text { gástrico }\end{array}$ & $N=261$ & $\begin{array}{l}\text { Pacientes identificados a } \\
\text { partir de um banco de dados } \\
\text { coletado prospectivamente } \\
\text { e enviados ao questionário } \\
\text { central da Organização } \\
\text { Européia para Pesquisa } \\
\text { e Tratamento do Câncer } \\
\text { (ELCR) (QLQ-C30 v.3) e } \\
\text { ao módulo gástrico (QLQ). } \\
\text {-STO22). } \\
\end{array}$ & $\begin{array}{l}\text { Não há diferença na QV } \\
\text { geral em pacientes com TG } \\
\text { ou SG, embora as restrições } \\
\text { alimentares e a disfagia } \\
\text { sejam piores após o TG. }\end{array}$ \\
\hline $\begin{array}{l}\text { YU, Wansik } \\
\text { et al. (2016) }\end{array}$ & $\begin{array}{l}\text { Coreia } \\
\text { do Sul }\end{array}$ & $\begin{array}{l}\text { Mudanças } \\
\text { Cronológicas } \\
\text { da Qualidade } \\
\text { de Vida em } \\
\text { Sobreviventes } \\
\text { após câncer } \\
\text { gástrico. }\end{array}$ & $N=254$ & $\begin{array}{l}\text { Foi utilizado dados de QV } \\
\text { da Organização Europeia } \\
\text { para Pesquisa e Tratamento } \\
\text { do Câncer QLQ-C30 no } \\
\text { pré operatório e } 1,2,3,4 \\
\text { e } 5 \text { anos após a cirurgia. } \\
\text { Utilizado também os } \\
\text { questionários QLQ-STO22. }\end{array}$ & $\begin{array}{l}\text { Não houve alteração } \\
\text { estatisticamente significativa } \\
\text { no estado de saúde global / } \\
\text { QV durante os } 5 \text { anos. }\end{array}$ \\
\hline
\end{tabular}


Os artigos apresentaram heterogeneidade em suas avaliações, no entanto é importante destacar que a associação da disfagia com o câncer gástrico tem sido estudada em escala crescente nos últimos anos, visando o bem estar do paciente e avaliando a melhor conduta clínica para os mesmos.

Dentre os resultados apresentados, observou-se a qualidade de vida de pacientes após câncer gástrico em três estudos incluídos como uma variável estudada. Adicionalmente, três estudos levam em considerações os diferentes tipos de tratamentos realizados do câncer gástrico que influenciam sobre a disfagia. Para alguns autores a qualidade de vida foi impactada positivamente, no entanto em outros estudos não foram observadas melhoras significativas que pudessem ser destacadas. No que se refere à disfagia, alguns autores destacam que existiu uma melhora no escore médio de disfagia, no entanto para outro estudo esse dado não foi efetivo.

\section{Discussão}

A relação entre o câncer gástrico e a disfagia tem sido abordada cientificamente como uma grande consequência dos vários tipos de tratamento. Três dos cinco estudos selecionados tratam da qualidade de vida dos pacientes posteriormente ao tratamento. $\mathrm{O}$ bem estar dos pacientes tem sido discutido em maior escala nos últimos anos, com o intuito de melhorar as condições do indivíduo que se encontra no estado de tratamento.

Park et. al (2014) selecionaram avaliar pacientes com câncer gástrico precoce, tendo como critério de exclusão pacientes com outros tipos de cânceres ou doenças que pudessem comprometer os resultados da pesquisa. No questionário de qualidade de vida, a disfagia foi abordada em módulo, juntamente com indicadores de dor, sintomas de refluxo, restrições alimentares e escalas de ansiedade, boca seca, paladar, imagem corporal e queda de cabelo. Destes, a disfagia foi o indicador que demorou mais tempo até a recuperação ${ }^{21}$.

Nos estudos de Goh et. al (2015) foram submetidos a pesquisa o total de 251 pacientes divididos em grupos pela forma de tratamento a qual foram submetidos, e notou-se que no caso de gastrectomia total a disfagia e as restrições alimentares foram muito maiores e mais impactantes na qualidade de vida pós tratamento ${ }^{22}$. Os pesquisadores indicam que o estudo demonstrou que não há diferença na qualidade de vida geral em pacientes com TG ou
SG, embora as restrições alimentares e a disfagia sejam piores após o TG.

Já o estudo de Yu et. al (2016) aponta mudanças cronológicas na qualidade de vida a longo prazo após gastrectomia, e demonstra que cinco anos após a cirurgia, a disfagia ainda se faz presente é um dos itens que ainda se faz presente na vida do paciente, ou seja afetam a longo prazo ${ }^{23}$. Destacam ainda que o tratamento dos sintomas gastrointestinais deve ser direcionado especificamente como parte do tratamento prolongado do paciente após uma gastrectomia. $\mathrm{O}$ cuidado nutricional adequado favorece a ingestão de alimentos, resultando em ganho de peso e melhor funcionamento físico, função funcional e imagem corporal.

Os estudos de Sunde et. al (2015) e Park et. al (2014) demonstraram a utilização de Stents Metálicos com objetivo de controle da disfagia nos casos de obstrução maligna em intervenções cirúrgicas decorrentes do câncer gástrico, onde se constatou uma melhora nos quadros de disfagia. Além do resultado, a pesquisa vem confirmar a relação e um dos efeitos do carcinoma gástrico na deglutição, levando à disfagia ${ }^{2324}$. A quimioterapia foi associada ao aumento da migração de stents e sobrevivência prolongada.

A literatura indica que estudos analisados indicam que a qualidade de vida pode ser influenciada pelo processo tumoral maligno, sendo induzidas situações como apoio familiar, tratamento utilizado, presença ou não de estomias dentre outros ${ }^{25}$. Reforçam também que a qualidade de vida de pacientes oncológicos, torna-se muito importante para verificar a eficácia e impactos de tratamentos e intervenções realizadas, além de se comparar procedimentos para o controle de morbimortalidades, planejar os melhores procedimentos e cuidados paliativos e detectar precocemente problemas emocionais e físicos. Todos estes aspectos corroboram com os estudos incluídos nesta pesquisa.

Segundo Silva (2007), técnicas terapêuticas e a eficácia da reabilitação em disfagia orofaríngea são estudas nas últimas décadas. Alguns estudos têm relatado a eficácia da reabilitação em disfagia orofaríngea, sendo mais frequente aqueles que têm se preocupado em comprovar os efeitos da técnica terapêutica na dinâmica da deglutição ${ }^{26}$.

Portanto, é possível notar uma relação entre o câncer gástrico e a disfagia confirmada pela literatura. Percebe-se que a disfagia pode estar 
diretamente relacionada com o tratamento ao qual o paciente foi submetido, impactando diretamente em sua qualidade de vida e, consequentemente, na nutrição.

\section{Conclusão}

Apesar do refinamento criterioso na busca por artigos, não foi possível recuperar estudos que tratam especificamente da associação da disfagia como uma consequência do carcinoma gástrico, sendo esta uma limitação dos estudos. Entretanto, é possível notar que na literatura muitos autores relatam a disfagia como um sintoma observado após o tratamento do câncer, levando a entender a alteração da qualidade de deglutição como uma consequência nos casos de neoplasias gástricas e está diretamente relacionada à forma de tratamento escolhida.

Notou-se que a principal forma de abordagem da disfagia nos estudos selecionados foi a partir de questionários feitos aos pacientes, onde apesar de eficientes ao refletir a percepção do afetado, não avaliam condições específicas da qualidade de deglutição, como ocorre em protocolos e testes exclusivos de deglutição, podendo avaliar de forma eficaz sinais clínicos ao risco de disfagia.

Além disso, verificou-se que não há um padrão de forma e tempo de tratamento entre os pacientes em cada estudo, o que dificultou a associação entre os casos, porém mostrou-nos a amplitude de casos e as diferenças entre eles.

Por fim, destaca-se que além da disfagia ser abordada, os estudos ainda a apresentam de forma superficial e apenas segundo a visão dos pacientes. Sugere-se a realização de estudos mais aprofundados em testes e avaliações quanto à relação e o impacto do câncer gástrico no processo de deglutição.

\section{Referências}

1. Instituto Nacional de Câncer José Alencar Gomes da Silva [homepage na internet]. Câncer de Estômago [ acesso em 09 set. 2018]. Disponivel em: https://www.inca.gov.br/tipos-decancer/cancer-de-estomago

2. Villaverde RM, Gordo AMJ, Moral SJ, Soto MAM. Cáncer de estómago. Medicine - Programa de Formación Médica Continuada Acreditado. Elsevier BV. 2017; 129(32): 1904-10.

3. Wagner AD; Syn NL, Moehler M, Grothe W, Yong WP, Tai $\mathrm{BC}$ et al. Chemotherapy for advanced gastric cancer. Cochrane Database Syst Rev. 2002; 8(2): 09-13.
4. Santos TE; Silva AHO; Caldeira LM. Frequência de câncer gástrico no serviço de endoscopia de um hospital-escola de Goiânia. GED -Gastronterologia Endoscopia Digestiva. 2016; 3(35): 89-95

5. Oliveira VA, Oliveira T W N, Alencar MVOB, Cerqueira, GS, Peron AP, Sousa JMC. Relação entre consumo alimentar da população nordestina e o alto índice de câncer gástrico nesta região. Revinter. 2014; 3(7): 6-24.

6. Pinto AS; Grigoletti SS; Marcadenti A. Fasting abbreviation among patients submitted to oncologic surgery: systematic review. Brazilian archives of digestive surgery. 2015; 28(1): 70-3.

7. Brito D, Raimundo A, Sousa O, Pereira H, Ribau E, Afonso LP et al. Recomendações para o diagnóstico e tratamento do adenocarcinoma gástrico. Revista Portuguesa de Cirurgia. 2014; 28(.2): 45-56.

8. Wilches RO, Navia HF, González CEB, Pedraza RS. Terapia de conversión en cáncer gástrico estado IV: a propósito de un caso. Rev Colomb Gastroenterol. 2018; 33(1): 61-72.

9. Laffitte AM; Polakowski CB; Kato M. Early Oral Re-Feeding On Oncology Patients Submitted To Gastrectomy For Gastric Cancer. Arquivos brasileiros de cirurgia digestiva. 2015; 28(3): 200-3.

10. Toneto MG; Viola L. Current Status Of The Multidisciplinary Treatment Of Gastric Adenocarcinoma. Arquivos brasileiros de cirurgia digestiva. 2018; 31(2): 43-52.

11. Ruivo EA, Fazeres FQ, Ventura J, Vasconcelos E, Terleira $\mathrm{H}$, Veiga $\mathrm{M}$ et al. Impacto do suporte nutricional precoce na morbimortalidade em doentes submetidos a cirurgia de resseção por adenocarcinoma gástrico. Revista Portuguesa de Cirurgia. 2015; 34(.3): 65-77.

12. Furkim AM, Santini CRQS. Disfagia Orofaringea. 2. ed. Barueri- Sp: Pró-fono, 2008. 237 p.

13. Almeida RCA; Haguette RCB; Andrade, ISN. Deglutição com e sem comando verbal: achados videofluoroscópicos. Revista da Sociedade Brasileira de Fonoaudiologia. 2011; 16(3): 291-7.

14. Zancan M, Luchesi KF; Mituuti CT, Furkim AM. Locais de início da fase faríngea da deglutição: meta-análise. CODAS. 2017; 29(2): 1-8.

15. Dragone MLS. Disfonia e disfagia: interface, atualização e prática clínica. Revista da Sociedade Brasileira de Fonoaudiologia. 2010; 15(4): 624-5.

16. Cola PC, Gatto AR; Silva RG; Schelp AO; Henry MACA. Reabilitação em disfagia orofaríngea neurogênica: sabor azedo e temperatura fria. CEFAC. 2008; 10(2):200-5.

17. Wegner DA, Steidl EMS, Pasqualoto AS, Mancopes R . Deglutição orofaríngea, nutrição e qualidade de vida no indivíduo com doença pulmonar crônica. CODAS. 2018; 30(3): 327-32.

18. Sassi F, Medeiros GC; Zambon LS, Zilberstein B, ECBCSP4, Andrade CRF. Avaliação e classificação da disfagia pósextubação em pacientes críticos. Revista do Colégio Brasileiro de Cirurgiões. 2018; 45(3): 200-9.

19. Moher D; Liberati A; Tetzlaff J; Altman DG, PRISMA Group. Preferred reporting items of systematic review and meta-analyses: the PRISMA statement. PLoS medicine. 2009; 7(7): 121-32. 
20. Park JY, Eom BW, Jo MJ, Yoon HM, Ryu KW, Kim YW et al. Health-Related Quality of Life After Robot-Assisted Distal Gastrectomy in Early Gastric Cancer. World Journal of Surgery. 2014; 38(5): 1112-20.

21. Goh YM, Gillespie C, Couper G, Paterson-Brown S. Quality of life after total and subtotal gastrectomy for gastric carcinoma. The Surgeon. 2015; 13(5): 267-70.

22. Yu W, Ki BP, Ho YC, Oh KK, Seung SL. Chronological Changes of Quality of Life in Long-Term Survivors after Gastrectomy for Gastric Cancer. Breast cancer research and treatment. 2016; 48(3); 1030-6.
23. Sunde B, Ericson J, Kumagai K, Lundell L, Tsai JA, Lindblad $\mathrm{M}$ et al. Relief of dysphagia during neoadjuvant treatment for cancer of the esophagus or gastroesophageal junction. Dis. Esophagus. 2015; 29(5): 442-7.

24. Park J, Song HY, Kim SH, Shin JH, Kim JH, Kim BS et al. Metallic Stent Placement in Patients with Recurrent Malignant Obstruction in the Surgically Altered Stomach. Annals of surgical oncology. 2014; 21(6): 2036-43.

25. Vieira AR, Fortes RC. Qualidade de vida de pacientes com câncer gastrointestinal. Com. Ciências Saúde. 2015; 26(1/2): 45-56.

26. Silva RG. A eficácia da reabilitação em disfagia orofaríngea. Pró-Fono R. Atual. Cient. 2007; 19(1): 123-33. 